\title{
Dentists welcome new national powers for water fluoridation
}

The Faculty of Dental Surgery and the Faculty of General Dental Practice UK have both welcomed proposals in the Government's new Health and Care White Paper, published on 11 February, which will give the Health Secretary new powers around water fluoridation. ${ }^{1}$

Responding to the proposals, Mr Matthew Garrett, Dean of the Faculty of Dental Surgery at the Royal College of Surgeons of England said: 'We welcome today's announcement as an important step forward in our efforts to improve children's oral health. Before the COVID-19 pandemic struck the UK, tooth decay was the leading cause of hospital admissions for five- to nine-year-olds by some distance, despite being entirely preventable. The events of the last 12 months, which have reduced access to dental services for many, will only have made this crisis worse. Water fluoridation has been found by Public Heath England to be an effective and safe measure for preventing dental decay. These proposals will make it easier for fluoridation schemes to be implemented more widely, so that more children can benefit'.

Ian Mills, Dean of the Faculty of General Dental Practice UK, added: 'Tooth decay is the most prevalent disease in England, and the provision of water which is either naturally or artificially fluoridated to one part per million is effective in reducing its incidence and severity. There is also clear evidence that it is a cost-effective approach which reduces the number of children admitted to hospital for tooth extractions, and that it offers the greatest benefit in more deprived areas which suffer from poorer oral health and wider health inequalities. However, only one in ten households in England currently receive tap water containing the recommended level of fluoride, and we support the proposals announced today, which would help reduce the barriers to offering fluoridated water to other communities which could benefit'.

The Government's Health and Care White Paper (p.61) proposes giving the 'Secretary of State for Health and Social Care the power to directly introduce, vary or terminate water fluoridation schemes. The Secretary of State for Health and Social Care already has the existing power to decide on whether proposals for water fluoridation should be approved and responsibility for the administration of schemes. This removes the burden from local authorities and will allow the Department of Health and Social Care to streamline processes and take responsibility for proposing any new fluoridation schemes, which will continue to be subject to public consultation. Central government will also become responsible for the associated work, such as the cost of consultations, feasibility studies, and the capital and revenue costs associated with any new and existing schemes.'

\section{Reference}

1. Department of Health and Social Care. Working together to improve health and social care for all. 11 February 2021. Available at: https://www.gov.uk/government/ publications/working-together-to-improve-health-andsocial-care-for-all (accessed 15 March 2021). 